\title{
ON A CONJECTURE OF HIGGINS
}

\author{
Philip R. Heath and Peter Nickolas
}

In his work on a proof of Grushko's theorem by groupoid methods, Philip Higgins proved the following subgroup theorem.

THEOREM . Suppose that $\theta: G \rightarrow B$ is a surjective map of groups, where $G$ and $B$ are free products $G=\prod_{\lambda}^{*} G_{\lambda}$ and $B=\prod_{\lambda}^{*} B_{\lambda}$, and where $\theta\left(G_{\lambda}\right)=B_{\lambda}$ for each $\lambda$. Let $H$ be a subgroup of $G$ with the property that the restriction of $\theta$ to $H$ is surjective. Then there is a free decomposition $\prod_{\lambda}^{*} H_{\lambda}$ of $H$ with $\theta\left(H_{\lambda}\right)=B_{\lambda}$ for each $\lambda$.

As Higgins has noted, there is a common strategy in the proof of this theorem and in the groupoid proofs of the Kurosh and Neilsen-Schreier subgroup theorems. Higgins conjectured the existence of a common generalisation of his theorem and the Kurosh theorem, proposed a plausible statement for such a result, and hinted that the common strategy of these proofs might be extended to give a proof of the conjecture. Examined at more detailed level, however, the common strategy used in the proofs of the Kurosh theorem and Higgins's theorem is seen to diverge into two strands. In this paper, it is shown that the constructions entailed in these divergent approaches are in general incompatible. Thus any proof of Higgins's conjecture must require substantially different techniques. In particular, the proof of a theorem by Ordman that purports to affirm the conjecture is incorrect, and the approach used in his argument cannot yield a valid proof.

\section{INTRODUCTION}

In his work on a proof of Grushko's theorem by groupoid methods, Philip Higgins $[3,4]$ proved the following subgroup theorem, from which Grushko's theorem may be deduced easily.

ThEOREM 1.1. ('Higgins's theorem') Suppose that $\theta: G \rightarrow B$ is a surjective map of groups, where $G$ and $B$ are free products $G=\prod_{\lambda}^{*} G_{\lambda}$ and $B=\prod_{\lambda}^{*} B_{\lambda}$, and where $\theta\left(G_{\lambda}\right)=B_{\lambda}$ for each $\lambda$. Let $H$ be a subgroup of $G$ with the property that the

Received 29th August, 1995

The first author thanks the Mathematical Analysis Research Group, Department of Mathematics, University of Wollongong, for its hospitality during the period of some of this work.

Copyright Clearance Centre, Inc. Serial-fee code: 0004-9729/96 \$A2.00+0.00. 
restriction of $\theta$ to $H$ is surjective. Then there is a free decomposition $\prod_{\lambda}^{*} H_{\lambda}$ of $H$ with $\theta\left(H_{\lambda}\right)=B_{\lambda}$ for each $\lambda$.

We note for reference and comparison the following detailed version of the Kurosh subgroup theorem (see [4] or [6], for example).

THEOREM 1.2. (The Kurosh subgroup theorem) Any subgroup $H$ of a free product $G=\prod_{\lambda}^{*} G_{\lambda}$ has a free decomposition $H=\prod_{\lambda \mu}^{*} H_{\lambda \mu} * F$, in which (i) for each $\lambda, H_{\lambda \mu}$ is of the form $H \cap x_{\lambda \mu} G_{\lambda} x_{\lambda \mu}^{-1}$, where, as $\mu$ varies, $x_{\lambda \mu}$ runs through a suitably chosen set of representatives of the double cosets $H x G_{\lambda}$, and (ii) $F$ is a free group.

Higgins has shown the utility of groupoid methods in combinatorial group theory by proving with a common strategy both the above theorems, and also the Nielsen-Schreier theorem [4]. In [4, p.125], Higgins asks whether one can strengthen the conclusion of Theorem 1.1 so that in the special case when $B$ is trivial it reduces to Theorem 1.2. $\mathrm{He}$ posits a plausible conjecture to this effect: that under the hypotheses of Theorem 1.1, the subgroup $H$ should have a free decomposition $H=\prod_{\lambda}^{*} H_{\lambda}$ in which for each $\lambda$ (i) $\theta\left(H_{\lambda}\right)=B_{\lambda}$, and (ii) $H_{\lambda}$ has a decomposition $H_{\lambda}=\prod_{\mu}^{*} H_{\lambda \mu} * F_{\lambda}$, where the $H_{\lambda \mu}$ are exactly as in Theorem 1.2 and $F_{\lambda}$ is free. In the last section of [3], Higgins hints-and it appears reasonable to hope-that the common strategy used for the above theorems might lead to a proof of the conjecture.

The strategy referred to above, and outlined by Higgins in [3], is in broad terms as follows: given a group $G$ and a subgroup $H$ of $G$, information about $G$ is lifted to the standard covering groupoid $\tilde{G}$ associated with $H$, which effectively has $H$ as vertex group; information about the structure of $H$ is then obtained by retracting $\tilde{G}$ onto the vertex group $H$ using a suitably chosen tree in $\widetilde{G}$. (See [4], and Section 2 below, for more details, including explanations of the terms.)

The precise location of the above tree within $\tilde{G}$, however, is intimately related to the details of the structural information that one obtains about $H$. In particular, the differing assertions made about $H$ by Higgins's theorem and by the Kurosh theorem place differing constraints on the location of the tree. In this paper we demonstrate that these constraints are in general incompatible. Specifically, we carefully analyse the situations in which the tree required for Higgins's theorem and the tree required for the Kurosh theorem are uniquely determined, and use this to show that it is not in general possible to find a tree satisfying both sets of constraints simultaneously. That is, a tree chosen so as to yield the conclusion of Higgins's theorem cannot at the same time yield the conclusion of the Kurosh theorem, and conversely. A consequence 
of this is that any proof of Higgins's conjecture must require substantially different techniques from those used in the existing groupoid proofs of both the above theorems. In particular the proof of the last part of Theorem A of Ordman [7], which if valid would affirm Higgins's conjecture, is incorrect. Indeed since Ordman uses techniques which are essentially equivalent to those of Higgins, the strategy of his proof cannot be used to obtain the claimed result. (We note, however, that Ordman's argument does correctly generalise Higgins's theorem to the case of an amalgamated free product.)

In Section 2, we establish our notation and briefly review some ideas from the theory of groupoids which are relevant here, and then discuss in some detail the subgroup theorem-proving strategy referred to above. Section 3 is devoted to the analysis outlined above.

\section{Preliminaries}

We assume that the reader has some familiarity with the theory of groupoids, and with the groupoid proofs of the Kurosh, Nielsen-Schreier and Grushko theorems (see [1] and [4], for example). We briefly summarise aspects of our notation and terminology which may not be standard.

If $G$ is a groupoid, we use the same symbol $G$ to denote the arrows (or edges) of $G$, and we write $\mathrm{Ob}(G)$ for the objects (or vertices) of $G$. If $g$ is an arrow of a groupoid, then $i(g)$ and $e(g)$ denote respectively the initial object and the terminal object (or end point) of $g$; we sometimes write $g: x \rightarrow y$, where $x=i(g)$ and $y=e(g)$.

If the groupoid $G$ is the free product [4] of subgroupoids $G_{\lambda}$, for $\lambda$ in some index set $\Lambda$, we write $G=\prod_{\lambda}^{*} G_{\lambda}$. If $F$ is the free groupoid [4] on a (directed) subgraph $\tau$, we write $F=F(\tau)$. We regard a group as a groupoid on a single object; with this convention, free products of groups and free groups are instances of the corresponding groupoid constructions, and we use the same notations for them.

We apply the term tree both to graphs and to groupoids. A (directed) graph $\tau$ is a tree graph if it is connected and has no cycles; a groupoid $T$ is a tree groupoid if it is connected and there is exactly one arrow from any one of its objects to any other. A groupoid generated by a tree subgraph is a tree groupoid, and is also the free groupoid on the subgraph.

If $G$ is any group and $H$ is any subgroup of $G$, then the standard covering [4] $\gamma: \widetilde{G} \rightarrow G$ induced by $H$ is defined as follows. The objects of $\widetilde{G}$ are the cosets $G / H$ of $H$ in $G$, and the arrows are the pairs of the form $(C, g)$, for $C \in G / H$ and $g \in G$. We have $i(C, g)=C$ and $e(C, g)=C g$, and the composition of arrows $(C, g)$ and $\left(C g, g^{\prime}\right)$ is $\left(C, g g^{\prime}\right)$. The covering morphism $\gamma$ is simply the projection from $\widetilde{G}$ to $G$. We note that $\widetilde{G}$ effectively has $H$ as its vertex group at the object $H$ : this vertex group is 
strictly speaking $\{(H, h): h \in H\}$, but it is frequently convenient to identify this with $H$ by implicitly applying the projection into the second coordinate.

Let $G$ be a connected groupoid, let $T$ be a wide tree subgroupoid in $G$, and let $V$ be a vertex group of $G$ at the vertex $v$. If for any vertex $w$ the unique arrow of $T$ from $v$ to $w$ is denoted by $t_{w}$, then the retraction induced by $T$ is the groupoid morphism $\rho: G \rightarrow V$ defined by $\rho(g)=t_{i(g)} g t_{e(g)}^{-1}$.

We now discuss in some detail the strategy described by Higgins in [3, p.372], and its use in the proofs of the Kurosh theorem and Higgins's theorem. For any group $G$ with subgroup $H$, let $\gamma: \widetilde{G} \rightarrow G$ be the standard covering described above. The first step in the groupoid proofs is to 'lift' structural information about $G$ to $\widetilde{G}$. Specifically, by [2] (see [4, Theorem 8]), if $G$ has the structure of a colimit, then that structure lifts in a natural way to a colimit structure on $\widetilde{G}$. As mentioned in the introduction, information about the structure of $H$ is then obtained by retracting $\tilde{G}$ onto $H$ using a suitably chosen tree in $\widetilde{G}$. As we also remarked earlier, however, the proof strategy diverges in the location of this tree. We now discuss this phenomenon in more detail.

We begin with the Kurosh theorem. Thus we are given that $H$ is a subgroup of a free product $G=\prod^{*} G_{\lambda}$. By [2], $\widetilde{G}$ can be written as a free product $\tilde{G}=\prod^{*} \widetilde{G}_{\lambda}$, where $\widetilde{G}_{\lambda}=\gamma^{-1}\left(G_{\lambda}\right)$. If we write the components of $\widetilde{G}_{\lambda}$ as $\widetilde{G}_{\lambda \mu}$ for some set of indices $\{\mu\}$ (which depends upon $\lambda$ ), then it is trivial that $\widetilde{G}_{\lambda}=\prod^{*} \widetilde{G}_{\lambda \mu}$. For each $\lambda$ and each $\mu$, we pick a vertex group $K_{\lambda \mu}$ of $\widetilde{G}_{\lambda \mu}$, together with a 'local' spanning tree graph $\tau_{\lambda \mu} \subseteq \widetilde{G}_{\lambda \mu}$. Now it is routine that each $\tau_{\lambda_{\mu}}$ is a free generating graph for the subgroupoid that it generates, as is the union $\bigcup_{\lambda \mu} \tau_{\lambda \mu}$, and so the above free decomposition of $\widetilde{G}$ may be refined to give

$$
\widetilde{G}=\prod_{\lambda \mu}^{*}\left(K_{\lambda \mu} * F\left(\tau_{\lambda \mu}\right)\right)=\prod_{\lambda \mu}^{*} K_{\lambda \mu} * F\left(\bigcup_{\lambda \mu} \tau_{\lambda \mu}\right)
$$

It is easy to see that $\bigcup \tau_{\lambda_{\mu}}$ spans $\widetilde{G}$, and we can therefore select a 'global' spanning tree graph $\tau \subseteq \bigcup_{\lambda \mu} \tau_{\lambda \mu}$. An argument which is not of direct relevance to us here (see [4, 1]) now implies that the retraction $\rho$ induced by the tree subgroupoid generated by $\tau$ preserves the free decomposition of $\widetilde{G}$ noted above, so that we have

$$
H=\prod_{\lambda \mu}^{*} \rho\left(K_{\lambda \mu}\right) * \rho\left(F\left(\bigcup_{\lambda \mu} \tau_{\lambda \mu} \backslash \tau\right)\right)=\prod_{\lambda \mu}^{*} \rho\left(K_{\lambda \mu}\right) * F\left(\rho\left(\bigcup_{\lambda \mu} \tau_{\lambda \mu} \backslash \tau\right)\right) .
$$

One confirms finally by routine algebra that the various factors on the right-hand side here are precisely those required in the conclusion of the Kurosh theorem.

We turn now to Higgins's theorem, and the proofs of it by both Higgins and Ordman. We note again that Ordman's argument in fact applies to the more general case of 
a free product with amalgamation. This extra generality is certainly of interest, but is essentially irrelevant to our discussion. We therefore ignore this aspect of Ordman's result, and discuss his proof as specialised to the case of an unamalgamated free product. We also ignore for the moment the detailed claims about the structure of the subgroup made in Ordman's Theorem A(8), though we shall return to these in the next section.

Thus we are given that $H$ is a subgroup of a free product $G=\prod^{*} G_{\lambda}$, that there is a surjection $\theta: G \rightarrow B=\prod^{*} B_{\lambda}$ with $\theta\left(G_{\lambda}\right)=B_{\lambda}$, and that the restriction of $\theta$ to $H$ is surjective. The essence of both Higgins's and Ordman's proofs $([7,4.1 .2],[4$, Theorem 12]) is the selection of a wide tree subgroupoid $T$ of $\widetilde{G}$ such that $T \subseteq$ ker $\theta \gamma$ and such that the retraction $\rho: \widetilde{G} \rightarrow H$ defined by $T$ preserves the decomposition $\widetilde{G}=\prod^{*} \widetilde{G}_{\lambda}$, so that $H \cong \prod^{*} \rho\left(\widetilde{G}_{\lambda}\right) \equiv \prod^{*} H_{\lambda}$; the fact that $T \subseteq \operatorname{ker} \theta \gamma$ then guarantees that $\theta\left(H_{\lambda}\right)=B_{\lambda}$, as required. We note that the arguments employed by Higgins and Ordman differ significantly (though they are perhaps equivalent in a suitable sense); but in both cases, the outcome is the construction of a tree $T$ with the properties mentioned above.

We can now describe more precisely how the strategies for Higgins's theorem and the Kurosh theorem diverge. Although in both cases the construction of a wide tree subgroupoid is the key step, in Higgins's theorem we have $T \subseteq \operatorname{ker} \theta \gamma$, while in the Kurosh theorem we have $T=F(\tau)$ for a wide tree graph $\tau \subseteq \bigcup \tau_{\lambda \mu}$. These constructions are in general incompatible, as we shall show.

\section{RESUlts}

Throughout this section $H$ will be a subgroup of a group $G$, and $\theta: G \rightarrow B$ a homomorphism with the property that the restriction of $\theta$ to $H$ is a surjection. Also $\gamma: \widetilde{G} \rightarrow G$ will be the standard covering of $G$ induced by $H$. We refer to any wide tree subgroupoid of $\widetilde{G}$ lying in $\operatorname{ker} \theta \gamma$ as a tree of type 1. (Our discussion of the proof of Higgins's theorem shows that the tree used there satisfies other important constraints in addition to being of type 1.)

If the restriction of $\theta$ to $H$ is one-to-one, then $\left.\theta\right|_{H}: H \rightarrow B$ is an isomorphism, and we may define a homomorphism $\eta: G \rightarrow H$ by setting $\eta=\left(\left.\theta\right|_{H}\right)^{-1} \circ \theta$. We note that $\theta(\eta(g))=\theta(g)$ for all $g \in G$.

We begin with two simple results.

Proposition 3.1. With the conventions of this section, there is a unique tree of type 1 if and only if the restriction of $\theta$ to $H$ is one-to-one.

Proof: Suppose that $\left.\theta\right|_{H}$ is one-to-one, so that $H \cap \operatorname{ker} \theta=\{1\}$. Clearly, for any $g \in G,\left(H, \eta\left(g^{-1}\right) g\right)$ is an arrow from $H$ to $H g$ lying in ker $\theta \gamma$. Let $\left(H, g_{1}\right)$ and $\left(H, g_{2}\right)$ lie in $\operatorname{ker} \theta \gamma$ and suppose that $H g_{1}=H g_{2}$. Then we have $g_{1} g_{2}^{-1} \in H \cap \operatorname{ker} \theta=\{1\}$, 
so $g_{1}=g_{2}$. Therefore there is precisely one arrow in $\operatorname{ker} \theta \gamma$ from the object $H$ to each other object of $\widetilde{G}$. It follows that there is precisely one wide tree subgroupoid of $\widetilde{G}$ contained in $\operatorname{ker} \theta \gamma$.

Conversely, if $\left.\theta\right|_{H}$ is not one-to-one, then $H \cap \operatorname{ker} \theta$ contains an element $g \neq 1$, so the vertex group at $H$ in $\widetilde{G}$ contains at least two elements of ker $\theta \gamma$. It follows that there are at least two arrows in $\operatorname{ker} \theta \gamma$ running between each pair of objects of $\widetilde{G}$. Therefore $\widetilde{G}$ contains more than one wide tree subgroupoid lying in ker $\theta \gamma$.

Proposition 3.2. Suppose that the restriction of $\theta$ to $H$ is one-to-one, and that $\eta$ is as above. Let $T$ be the unique tree of type 1 given by Proposition 3.1.

(i) The unique arrow in $T$ from $H x$ to $H x w$, for $x, w \in G$, is $\left(H x, x^{-1} \eta\left(x w^{-1} x^{-1}\right) x w\right)$. In particular, the unique arrow in $T$ from $H$ to $H w$, for $w \in G$, is $\left(H, \eta\left(w^{-1}\right) w\right)$.

(ii) If $w \in \operatorname{ker} \theta$ in (i), then $\left(H x, x^{-1} \eta\left(x w^{-1} x^{-1}\right) x w\right)=(H x, w)$.

(iii) Let $\rho$ be the retraction associated with $T$. Then for any $(H x, w) \in \tilde{G}$ we have $\rho(H x, w)=\eta(w)$. In particular, if $h \in H$, then $\rho(H x, h)=h$.

ProOF: For (i), we note that $H x x^{-1} \eta\left(x w^{-1} x^{-1}\right) x w=H \eta\left(x w^{-1} x^{-1}\right) x w=H x w$, since $\eta$ maps into $H$, and so $\left(H x, x^{-1} \eta\left(x w^{-1} x^{-1}\right) x w\right)$ is an arrow from $H x$ to $H x w$. But by the property of $\eta$ noted above, we also have $\left(H x, x^{-1} \eta\left(x w^{-1} x^{-1}\right) x w\right) \in \operatorname{ker} \theta \gamma$. For (ii), we note that both the arrows in question run from $H x$ to $H x w$, and that both lie in $\operatorname{ker} \theta \gamma$; since there is precisely one arrow with these two properties, the arrows are equal. Finally, using the fact that $\eta$ is a homomorphism, (iii) follows directly from (i) and the definition of $\rho$.

A situation of interest below is when a subgroup $H$ of a free product $\prod^{*} G_{\lambda}$ 'has no free factor'. This phrase, however, is ill-defined in general, since free factors of $H$ may in general arise not only as (factors of) the factor $F$ in the Kurosh theorem, but also as (factors of) conjugates of subgroups of the $G_{\lambda}$. When we wish to discuss subgroups with no free factor, we will therefore confine ourselves to cases where the $G_{\lambda}$ are torsion groups, and therefore have no non-trivial free subgroups. In this case it is not hard to see, by the results of [5, Chapter IX], for example, that the phrase above is well-defined: if a subgroup $H$ of $\prod^{*} G_{\lambda}$ has no (non-trivial) free factor in some free decomposition, however obtained, then the same is true for all free decompositions of $H$.

In the following propositions, we use the notation introduced earlier in the discussion of the proof of the Kurosh theorem. As we have already noted in that discussion the proof requires us to choose a tree graph $\tau \subseteq \bigcup \tau_{\lambda \mu}$. The first proposition states roughly that we need the whole of $\bigcup \tau_{\lambda \mu}$ to form $\tau$ precisely when there is no free factor. 
Proposition 3.3. Let $H$ be a subgroup of $\prod^{*} G_{\lambda}$, where the $G_{\lambda}$ are torsion groups. Then the following are equivalent:

(i) $H$ has no free factor;

(ii) for any choice of local tree graphs $\tau_{\lambda \mu} \subseteq \widetilde{G}_{\lambda \mu}$, the graph $\tau=\bigcup_{\lambda \mu} \tau_{\lambda \mu}$ is a spanning tree subgraph of $\widetilde{G}$.

Proof: We recall from the discussion in Section 2 that, for a given choice of the $\tau_{\lambda \mu}$, and of $\tau \subseteq \bigcup_{\lambda \mu} \tau_{\lambda \mu}$, the free factor in the decomposition of $B$ is given by $F\left(\rho\left(\bigcup_{\lambda \mu} \tau_{\lambda \mu} \backslash \tau\right)\right)$, where $\rho$ is the retraction defined by $\tau$. Given that the notion of having no free factor is well defined, it is then clear that $H$ has no free factor exactly when $\bigcup_{\lambda \mu} \tau_{\lambda \mu} \backslash \tau$ is empty, and this is exactly when $\bigcup_{\lambda \mu} \tau_{\lambda \mu}$ is itself a wide tree subgraph of $\tilde{G}$.

The proof of the following result is straightforward.

PROPOSITION 3.4. Given a subgroup $H$ of a free product $\prod^{*} G_{\lambda}$, the vertex group at the object $H x$ in $\widetilde{G}_{\lambda}$ is $\left\{(H x, g): g \in x^{-1} H x \cap G_{\lambda}\right\}$; hence the component of $\widetilde{G}_{\lambda}$ containing $H x$ is an isolated vertex group if and only if $H \cap x G_{\lambda} x^{-1}=x G_{\lambda} x^{-1}$, and is a tree groupoid if and only if $H \cap x G_{\lambda} x^{-1}=\{1\}$.

For any choice of local tree subgraphs $\tau_{\lambda \mu} \subseteq \widetilde{G}_{\lambda \mu}$ and of global tree subgraph $\tau \subseteq \bigcup \tau_{\lambda \mu}$, the subgroupoid of $\widetilde{G}$ generated by $\tau$ is freely generated, and is a wide tree subgroupoid of $\tilde{G}$. We refer to any wide tree subgroupoid constructed in this way as a tree of type 2 .

Proposition 3.5. Let $H$ be a subgroup of $\prod^{*} G_{\lambda}$, where the $G_{\lambda}$ are torsion groups. Then there is a unique tree of type 2 in $\widetilde{G}$ if and only if (a) for each $\lambda$ and for every $x \in G, H \cap x G_{\lambda} x^{-1}$ is either $x G_{\lambda} x^{-1}$ or $\{1\}$, and (b) $H$ has no free factor.

Proof: First, suppose that (a) and (b) hold. Suppose that local spanning tree graphs $\tau_{\lambda \mu}$ are selected in the components $\widetilde{G}_{\lambda \mu}$. By Proposition 3.3, the choice of a global spanning tree subgraph $\tau \subseteq \bigcup \tau_{\lambda \mu}$ is unique, and we must in fact have $\tau=\bigcup \tau_{\lambda \mu}$.

Let $T$ denote the tree groupoid freely generated by $\tau$, and let $T_{\lambda \mu}$ denote the tree groupoid freely generated by $\tau_{\lambda \mu}$, for all $\lambda$ and $\mu$. Then $T$ clearly contains $\bigcup_{\lambda \mu} T_{\lambda \mu}$, and therefore contains the subgroupoid $F$ generated by $\bigcup_{\lambda \mu} T_{\lambda \mu}$. But $T$ is also clearly contained in $F$, so we have $T=F$. Now suppose that another choice of local spanning tree graphs is made-say $\tau_{\lambda \mu}^{\prime}$. Defining $T_{\lambda \mu}^{\prime}, T^{\prime}$ and $F^{\prime}$ analogously, we then have $T^{\prime}=F^{\prime}$, as above. But $\tau_{\lambda \mu}$ and $\tau_{\lambda \mu}^{\prime}$ generate the same subgroupoids (for all $\lambda$ and 
$\mu$ ), since all components of $\widetilde{G}_{\lambda}$ are isolated vertex groups or tree groupoids, by (a) and Proposition 3.4. That is, $T_{\lambda \mu}=T_{\lambda \mu}^{\prime}$ for all $\lambda$ and $\mu$; and it follows that $F=F^{\prime}$, and therefore that $T=T^{\prime}$. Thus there is a unique tree of type 2 .

Second, suppose that (a) is false. Then by Proposition 3.4, there are $\lambda$ and $\mu$ such that $\tilde{G}_{\lambda \mu}$ has distinct vertices $C_{1}$ and $C_{2}$ for which there are distinct edges $\tilde{g}, \tilde{g}^{\prime}: C_{1} \rightarrow C_{2}$. Now we may select a local tree $\tau_{\lambda \mu}$ in $\widetilde{G}_{\lambda \mu}$ containing $\tilde{g}$, and another local tree $\tau_{\lambda \mu}^{\prime}$ containing $\tilde{g}^{\prime}$. Given choices of all other local trees, we may then choose global tree subgraphs $\tau$ and $\tau^{\prime}$, the first containing $\tilde{g}$, the second containing $\tilde{g}^{\prime}$. Then the tree subgroupoids $T$ and $T^{\prime}$ of type 2 which these graphs generate are distinct, since each has precisely one edge from $C_{1}$ to $C_{2}$, and $T$ contains $\tilde{g}$, while $T^{\prime}$ contains $\tilde{g}^{\prime}$.

Finally, suppose that (b) is false, so that $H$ has a non-trivial free factor. Then Proposition 3.3 shows that, for some choice of local trees $\tau_{\lambda \mu}$, the graph $\bigcup \tau_{\lambda \mu}$ is not a tree. Choose a global tree subgraph $\tau \subseteq \bigcup \tau_{\lambda \mu}$. Now $\tau$ and $\bigcup \tau_{\lambda \mu}$ both freely generate the subgroupoids that they generate, so it follows that the tree subgroupoid $T$ generated by $\tau$ is a strict subgroupoid of that generated by $U \tau_{\lambda_{\mu}}$. Therefore there is an edge $t \in \bigcup \tau_{\lambda_{\mu}}$ such that $t \notin T$. Hence it is possible to choose a global tree subgraph $\tau^{\prime} \subseteq \bigcup \tau_{\lambda \mu}$ containing $t$, and $\tau^{\prime}$ generates a tree $T^{\prime}$ of type 2 distinct from $T$.

Thus we have, in Propositions 3.1 and 3.5, simple necessary and sufficient conditions for the uniqueness of trees of type 1 and type 2. Using these criteria, we are able to discuss classes of examples in a generic fashion. We use them to demonstrate the incompatibility of the two proof strategies discussed in Section 1.

EXAMPLE 3.6. Let $G_{1}, G_{2}$ and $G_{3}$ be non-trivial torsion groups and set $G=$ $G_{1} * G_{2} * G_{3}$. Also, let $B=G_{1} * G_{2} *\{1\}$ and let $\theta: G \rightarrow B$ be the natural projection. Let $g_{2} \in G_{2} \backslash\{1\}$ and $g_{3} \in G_{3} \backslash\{1\}$ be fixed. We consider the subgroup $H=g_{2} G_{1} g_{2}^{-1} * g_{3} G_{2} g_{3}^{-1}$ of $G$.

It is clear by Propositions 3.1 and 3.5 that the covering groupoid $\widetilde{G}$ contains a unique tree subgroupoid $T_{1}$ of type 1 and a unique tree subgroupoid $T_{2}$ of type 2 .

Let the local trees $\tau_{i \mu}$ (for $i=1,2,3$ and for all $\mu$ ) be chosen, but arbitrary. By the uniqueness of $T_{2}$ and Proposition 3.3, $T_{2}$ must contain all the $\tau_{i \mu}$, and therefore also the subgroupoids which these generate. Now the component of $\widetilde{G}_{2}$ containing the coset $H$ is a tree, since $H \cap G_{2}=\{1\}$, and has as vertices exactly the cosets $H g$, for $g \in G_{2}$. Therefore $T_{2}$ must contain all the edges $(H, g)$ for $g \in G_{2}$. But none of these, except $(H, 1)$, is in $T_{1}$, and we therefore have the following result.

PRoposition 3.7. In Example 3.6, $T_{1} \neq T_{2}$.

Thus Proposition 3.7 confirms our claim that it is not in general possible to find 
a tree satisfying simultaneously the constraints required to yield the conclusion of Higgins's theorem and the conclusion of the Kurosh theorem. By the argument above, we see in particular that $T_{2}$ contains the edge $\left(H, g_{2}\right)$, and since the vertex group at the coset $H g_{2}$ is clearly $\left\{\left(H g_{2}, g\right): g \in G_{1}\right\} \cong G_{1}$, we see that one of the factors appearing in the decomposition of $H$ generated by the proof of the Kurosh theorem is precisely $g_{2} G_{1} g_{2}^{-1}$. Similarly, we find that $g_{3} G_{2} g_{3}^{-1}$ appears as a factor, and since there are no other conjugates of $G_{1}, G_{2}$ and $G_{3}$ which $B$ intersects non-trivially, and since $H$ has no free factor, we have the following.

Proposition 3.8. In Example 3.6, the groupoid proof of the Kurosh theorem yields precisely the originally given free decomposition of $H$, regardless of how the local tree subgraphs are chosen.

REMARK 3.9. Since $\theta\left(g_{2} G_{1} g_{2}^{-1}\right) \neq G_{1}$, this decomposition of $H$ does not 'lie over' the factors of $B$. Higgins' theorem, however, implies that there is a decomposition of $H$ which does. It is easy to see that this is the decomposition

$$
H=\left(g_{3} g_{2}^{-1} g_{3}^{-1}\right) g_{2} G_{1} g_{2}^{-1}\left(g_{3} g_{2}^{-1} g_{3}^{-1}\right)^{-1} * g_{3} G_{2} g_{3}^{-1} .
$$

Proposition 3.8 shows that this decomposition of $H$ cannot arise by the construction in the proof of the Kurosh theorem.

It remains to show that our analysis also provides a simple construction of a counterexample to the claims made in Ordman's Theorem A(8). We could use Example 3.6 again to demonstrate this, but we use a different example for simplicity.

EXAMPLE 3.10. Let $G_{1}$ and $G_{2}$ be non-trivial torsion groups and set $G=G_{1} * G_{2}$. Also, let $B=G_{1} *\{1\}$ and let $\theta: G \rightarrow B$ be the natural projection. We consider the subgroup $H=G_{1}$ of $G$. (Though $H$ and $G_{1}$ are the same group, it is convenient to refer to this group as $H$ when focusing on its role as a subgroup, and as $G_{1}$ when focusing on its role as a factor of the free product $G$.)

We shall show below that, when Ordman's proof is specialised to this example, the subgroup $H$ is given a non-trivial free factor, whose set of free generators includes all the members of the set $G_{1} \backslash\{1\}$. This is clearly impossible.

By Proposition 3.1, it is easy to see that in Example 3.10 there is once again a unique tree subgroupoid $T_{1}$ of type 1 . Because of its uniqueness, $T_{1}$ must be the tree described in Ordman's proof, specialised to our example. Also, by Proposition 3.4, each component $\widetilde{G}_{i \mu}$ (for $i=1,2$ and for all $\mu$ ) is either an isolated vertex group or a tree groupoid. Select in each $\widetilde{G}_{i \mu}$ an arbitrary vertex $B_{i \mu}$ as 'basepoint', and let $\sigma_{i \mu}$ be the unique spanning tree graph of $\widetilde{G}_{i \mu}$ containing an edge from $B_{i \mu}$ to every other vertex in $\widetilde{G}_{i \mu}$. Let $\rho_{1}$ denote the retraction defined by $T_{1}$. Then Ordman's proof of Theorem 
$A(8)$ asserts (using our notation) that the non-trivial elements in the set

$$
\rho_{1}\left(\bigcup_{\mu} \sigma_{1 \mu} \cup \bigcup_{\mu} \sigma_{2 \mu}\right)
$$

form a free generating set for a free factor of $H$ (see Proposition 4.4.3, and the first paragraph of 4.3 , of [7]). We show that this set contains $G_{1} \backslash\{1\}$.

To this end, consider a component of $\widetilde{G}_{1 \mu}$ containing a coset $H x \neq H$. Since $H x \neq$ $H$, and the only non-trivial intersection of $H$ with a conjugate of $G_{1}$ is $H$ itself, we see by Proposition 3.4 that the component we are considering is a tree groupoid. Suppose that the basepoint selected is $H x g_{0}$, for some $g_{0} \in G_{1}$. Then the set of purported free generators contains the non-identity elements of $\rho_{1}\left(\left\{\left(H x g_{0}, g_{0}^{-1} g\right): g \in G_{1}\right\}\right)$. Now using Proposition 3.2 (iii) we find that, for $g \in G_{1}, \rho_{1}\left(\left(H x g_{0}, g_{0}^{-1} g\right)\right)=g_{0}^{-1} g$, and so the set contains the elements $g_{0}^{-1} g$ for all $g \in G_{1} \backslash\left\{g_{0}\right\}$; that is, the elements of the set $G_{1} \backslash\{1\}$. This, as noted above, is impossible.

REMARK 3.11. As we claimed earlier, the argument above shows not just that Ordman's proof is incorrect, but that the strategy of his proof cannot be used to obtain the claimed result about the fine structure of the subgroup $H$. As Proposition 3.7 demonstrates, a tree chosen to yield a decomposition of $H$ lying over the factors of $B$ cannot in general also yield the claimed fine decomposition of $\boldsymbol{H}$.

REMARK 3.12. The error in the proof of Theorem A(8) appears to lie in Ordman's Lemma 4.4.2. If $g_{1}$ and $g_{2}$ are arbitrary non-trivial elements of $G_{1}$ and $G_{2}$, respectively, then the product

$$
c_{1} q_{1} c_{2} q_{2} \equiv\left(H, g_{1}\right)^{-1}\left(H, g_{2}\right)\left(H g_{2}, g_{1}\right)\left(H, g_{1}^{-1} g_{2} g_{1}\right)^{-1}
$$

(using Ordman's notation on the left and ours on the right) is easily seen, using Proposition 3.2 (ii), to contradict the assertion of that lemma.

\section{REFERENCES}

[1] R. Brown, Topology: a geometric account of general topology, homotopy types and the fundamental groupoid (Ellis Horwood/Simon and Schuster, 1988).

[2] P.R. Heath and K.H. Kamps, 'Lifting colimits of (topological) groupoids and (topological) categories', in Categorical topology and its relation to analysis, algebra and combinatorics (Prague, 1988) (World Scientific Publishing, New Jersey, 1989), pp. 54-88.

[3] P.J. Higgins, 'Grushko's theorem', J. Algebra 4 (1966), 365-372.

[4] P.J. Higgins, Notes on categories and groupoids (Van Nostrand Reinhold, London, 1971).

[5] A.G. Kurosh, The theory of groups, Vol. II, (English translation) (Chelsea, 1956). 
[6] S. MacLane, 'A proof of the subgroup theorem for free products', Mathematika 5 (1958), 13-19.

[7] E.T. Ordman, 'On subgroups of amalgamated free products', Proc. Camb. Phil. Soc. 69 (1971), 13-23.

Department of Mathematics

Memorial University of Newfoundland

St. John's

Newfoundland A1C 5S7

Canada

\author{
Department of Mathematics \\ University of Wollongong \\ Wollongong NSW 2522 \\ Australia
}

